tive decline during that period of the optical glass manufacture in this country and the consequent absence of an active and close local liaison between the English mathematicians and opticians on one hand and the optical glass manufacturers on the other? The very presence in a country of an optical glass factory focusses interest on, and enlarges the conceptions of, the research problems connected with optical glass and its applications to optical instruments. The mathematicians and optical designers who are to open out new paths of advance should have the materials they need at hand and readily available. To allow the optical glass industry to die out in this country would mean that not only the spirit of invention in this industry, but also much of that spirit in the dependent industry of optical instrument manufacture, would pass over to the country in which there was close co-operation between these essentially related industries.

Messrs. Zeiss assert that the practical monopolisation of the glass market by French and English houses between 1848 and 1883 materially hampered the progress of the optical engineer. What reason have we to think that a similar monopolisation to-day by Germany would be less detrimental to the development of the optical instrument industry in this country? If Germany were the only source of supply of optical glass, what guarantee have we that preferential treatment would not be given to German optical manufacturers in matters of time, quality, and quantity, to the prejudice of optical manufacturers of other nations? Would the British engineering industry have reached its present excellence if there had been no efficient and vigorous iron and steel industry in this country, and consequently no continuous and intimate co-operation on the spot between the engineer and the iron and steel manufacturer? These considerations do not, of course, apply to all industries. But the optical instrument industry depends, and must depend, on constant and close co-operation and co-ordination between the optician and the mathematician on one hand and the manufacturer of optical glass on the other, and this cannot be complete and efficient in a country where the sole source of optical glass is a foreign supply.

We agree with Messrs. Zeiss's concluding statement that optical mathematicians of all countries need every extension of the choice of glasses at their disposal and would deplore their exclusion from any valuable material available to opticians in foreign countries. But there was no proposal in the article in NATURE of February Io last designed or bound to have this effect, and, in any case, it leaves untouched the argument for maintaining, in the interests of British opticians, a healthy and progressive optical glass industry in this country.

The Writer of the Article.

\section{The Tendency of Elongated Bodies to set in the North and South Direction.}

AT one of the soirées of the Royal Society in 1920, Mr. A. E. Reeves showed an apparatus by means of which he believed he had obtained evidence that under suitable atmospheric conditions freely suspended elongated bodies set themselves with their longer axes in the geographical meridian. The evidence supplied at the time was not very convincing, and I understand that the subject is receiving further attention. In the meantime it may be pointed out that the earth's centrifugal force would act in a manner tending in the direction of the alleged effect, though the resulting couple is so minute that it would be extremely difficult to verify it experimentally.

If a horizontal rod be placed in the north and south direction, its southern end is-in the northern hemisphere-further away from the earth's axis. The centrifugal force is therefore greater at the southern end, and if the rod be slightly displaced, the horizontal component of that force will tend to bring the rod back into the meridian plane.

If $\rho_{0}$ be the distance of the centre of the rod from the earth's axis, that of a point at a distance $s$ from the centre will be $\rho=\rho_{0}+s \cos \theta$, where $\theta$ is the $\operatorname{co}$ latitude.

The horizontal component of the centrifugal force per unit mass at any point of the rod is $\omega^{2} \rho \cos \theta$. It is obvious that only the variation of the centrifugal force along the rod can produce an effect, so that we may write for its significant part $\omega^{\prime} s \cos \theta$. If the rod be turned through an angle $\phi$ we must apply a further factor $\cos \phi$, neglecting small quantities of the second order. With $\sigma$ for the mass per unit length of the rod, the couple acting on it becomes

$$
\int \sigma \omega^{2} s \cos ^{2} \theta \sin \phi \cos \phi d s=\mathrm{I} \omega^{2} \cos ^{2} \theta \sin \phi \cos \phi,
$$

where $I$ is the moment of inertia of the rod about its centre of inertia. The result will be the same far any lamina, whatever its shape or material. For small values of $\phi$ the vibrations of the rod are determined by :-

$$
\mathrm{I} \frac{d^{2} \phi}{d t^{2}}=\mathrm{I} \omega^{2} \cos ^{2} \theta \cdot \phi
$$

which gives the period of a complete oscillation as independent of $\mathrm{I}$ and equal to $2 \pi / \omega \cos \theta$, or $\mathrm{T} \sec \theta$, if $\mathrm{T}$ be the time of revolution of the earth. We find, therefore, that the suspended body tends to perform oscillations round the meridian position, the time of a complete oscillation being the same as that which Foucault's pendulum requires to turn round a complete circle, which in our latitude is about $3 \mathbf{I}$ hours.

The maximum torsional couple takes place when the rod is inclined at an angle of $45^{\circ}$ to the meridian, and in a latitude of $45^{\circ}$ it is $\frac{1}{4} \mathrm{I} \omega^{2}$. If it be desired to demonstrate it experimentally we should naturally turn to quartz fibres on account of their great carry. ing power. According to Sir Richard Threlfall (Phil. Mag., vol. 30, p. 99, 1890), a quartz fibre $0.001 \mathrm{~cm}$. in diameter can carry about ro grams. A uniform rod of that weight and $30 \mathrm{~cm}$. in length has a moment of inertia $75^{\circ}$. By a suitable distribution of the weight of the rod this might be increased to Iooo. The numerical value of the resulting couple then becomes

$$
250 \omega^{2}=\mathrm{r} \cdot 3 \times 10^{-6} .
$$

For the couple due to a unit angular torsion of a quartz thread of unit length and radius $r$, Sir Richard Threlfall gives $4.7 r^{4} \times 10^{11}$. If the length of the thread be $47 \mathrm{~cm}$., we find finally $\mathrm{r} \cdot 3 \times 10^{-4}$, or about 20 seconds of arc for the angular torsion of the thread which balances the couple due to centrifugal force. I believe that the finest threads have a diameter about ten times smaller than that in the example given, but the weights would then have to be divided by 10o, and the angular displacement would be between three and four minutes of arc. It is to be noted further that the effect cannot be observed directly because we cannot remove or apply the centrifugal force at will; the whole apparatus would therefore have to be turned through an angle of $45^{\circ}$, and the difference measured between that angle and the angular displacement of the suspended body. ARTHur SCHUSTER.

NO. 2712 , VOL. IO8] 